\title{
Chronobiological data: advantages of telemetric monitor- ing and prospects of mathematical modeling
}

\author{
Mikhail L. Blagonravov ${ }^{1, *}$, Björn Lemmer ${ }^{2, * *}$, Anna A. Bryk ${ }^{1, * * *}$, Vyacheslav A. \\ Goryachev $^{1, * * * *}$, Abdukayum A. Karimov ${ }^{1, \dagger}$, and Sergey M. Chibisov ${ }^{1, \ddagger}$ \\ ${ }^{1}$ Peoples' Friendship University of Russia (RUDN University) \\ ${ }^{2}$ Medical Faculty Mannheim University of Heidelberg
}

\begin{abstract}
Chronobiology and chronomedicine is a special part of biomedical sciences studying rhythmical patterns in physiological and pathological processes. In order to analyse probability of some pathology and to make forecasts concerning possibility of some diseases based on the signs of rhythmicity disorders it is necessary to perform continuous monitoring of different physiological functions for a certain period of time. Since 1984 until now we have had an excellent opportunity of studying biological rhythms and their disorders in animals using the method of radio-telemetric monitoring. A huge amount of continuous data obtained in telemetric monitoring could be used for mathematical modeling of different pathological processes on the basis of rhythmic patterns. In this work we have presented some preliminary results of the chronobiological study in which the effects of bright light on blood pressure and heart rate were investigated. The experiment was carried out on male rats of genetic strains: Wistar-Kyoto - normotensive rats and SHR - spontaneously hypertensive rats. The animals were exposed to 1 hour exposure of $\sim 10000$ lux white LED light from 10.00 to $11.00 \mathrm{a} . \mathrm{m}$. For the analysis of daily profiles of blood pressure and heart rate we used the method of radio-telemetric monitoring of blood pressure and heart rate. It was shown that systolic blood pressure significantly increased in both Wistar-Kyoto and SHR rats under the action of bright during the time of bright light exposure (from 10.00 to $11.00 \mathrm{a} . \mathrm{m}$.) and within the whole daytime period. For SHR rats an increase in diastolic blood pressure during the period of bright light action was also typical. But there were no significant changes in heart rate in the animals of either strain. These results require further and more detailed chronobiological studies to provide additional evidence. However traditional statistical methods seem to be important but not sufficient for further investigations. Moreover we could lose a considerable part of data without using contemporary methods of computer and mathematical modeling.
\end{abstract}

\footnotetext{
*e-mail: blagonravovm@mail.ru

**e-mail: bjoern.lemmer@medma.uni-heidelberg.de

***e-mail: bryckanna@gmail.com

****e-mail: goryachev-va@rudn.ru

†e-mail: abdukayum.karimov@bk.ru

†e-mail:kalcna@mail.ru
} 


\section{Introduction}

Chronobiology and chronomedicine as an independent part of Life sciences began to take shape relatively recently - in the 50-s of XX-th century, although the first mention of the cyclical nature of the onset and exacerbations of various diseases (primarily infectious) dates back to the time of Hippocrates.

In 1950s the concept of circadian (about-24-hour) rhythms - the main biological rhythms of living matter was formulated. In 1959 Franz Halberg (University of Minnesota) suggested the term "circadian" to describe about 24-hour rhythms [4]. In 1984 Jürgen Aschoff together with his colleagues from the United States, Britain, Germany, the Netherlands published a monograph "Biological rhythms". In this works J. Aschoff showed that it is light that stabilizes the circadian rhythms of the body [2].

Biological rhythms are a fundamental property of the living matter. Life without rhythm is impossible. Rhythmic activity is characteristic at different levels of organization of life: form unicellular to multicellular organisms. Along with the studies of normal biological rhythms, numerous investigations have shown that the pathogenesis of a number of diseases is closely related to the desynchronization of biological rhythms which is understood as loss of synchronization of internal and external rhythms which were previously synchronized.

\section{Research methods used in chronobiology and chronomedicine}

The principle of studying biological rhythms is different from the traditional approaches used in biomedical research. In this case it is necessary to use the monitoring technique of various body functions over a certain period of time ( 1 hour, 24 hours, several days, etc.). This makes it possible to assess the patterns of fluctuations of some parameters over time.

The methods such as ambulatory blood pressure monitoring (ABPM) and Holter monitoring of ECG were introduced into clinical practice long time ago. Using these methods, we obtain at least the results of 84 measurements of blood pressure (with ABPM) and a continuous ECG presentation (with Holter monitoring) within a 24-hour period. However disorders of biological rhythms commonly develop prior to the onset of some disease and may have a latent character.

In order to analyse probability of some pathology and to make forecasts concerning possibility of some diseases based on rhythmicity disorders it is necessary to perform continuous monitoring of different physiological functions for a given period of time which is sometimes impossible in clinical practice.

\subsection{Radio-telemetry monitoring}

1991 was an important milestone in the development of chronobiology, when the world's first wireless radio-telemetry monitoring technique was proposed [1]. From 1991 until now, we have had the opportunity to study biological rhythms and their disturbances in an animal experiment using the telemetry monitoring technique. The essence of this technique is that miniature devices are implanted in animals and measure blood pressure, heart rate, ECG, motor activity, body temperature and additional functions and to transmit information as a radio-signal to the receiving devices and storing data in the computer. Professor Björn Lemmer (Heidelberg University) was the first to introduce this method in experimental chronopharmacology in Europe [6].

The main advantages of telemetric monitoring in comparison with other research methods used to study the functions of organs and systems are as follows: 
1. Continuous 24-hour monitoring of some parameters/functions for at least several months.

2. During the experiment animals are unrestrained and totally free moving. They do not suffer from pain, they are not stressed, they are not affected by anaesthesia.

3. We perform not just separate measurements of blood pressure, heart rate, body temperature and other indicators but continuous monitoring of these functions.

4. The experiments only need small number of animals to be valid.

5. Animals are not sacrificed due to the study.

The telemetry system consists of a radio transmitter (which is implanted into the animal's body during a surgical operation), a receiver (takes the radio-signal from transmitters) and a computer (saving data on the hard drive) $[1,6]$.

As a result, we get a huge array of data that can be analyzed using various statistical methods used in chronobiology and chronomedicine.

\subsection{Programs and methods}

In our investigations we commonly use the computer program "Chronos-Fit" which performs Rhythm Analysis as a combination of partial Fourier analysis and a stepwise regression technique. Rhythm analysis fits each harmonic separately and checks significance by F-test for each [7].

Other methods such as Cosinor Analysis or Wavelet Transform can serve as good tools but they also have some restrictions. At the same time a huge amount of continuous data obtained in telemetric monitoring could be used for mathematical modeling of different pathological processes on the basis of rhythmic patterns.

\section{Some preliminary results}

In the recent years attention is being increasingly focused on the problem of improper use (misuse) of artificial light. On the one hand the great majority of people all over the world lead their activity under artificial light patterns which are mainly characterized by a prolonged duration of daylight. Also "light pollution" which is characterized by excessive exposure of light in the night time is still a problem of great concern. Both these factors may induce the development of desynchrony - a complex of disorders caused by desynchronization of biological rhythms. On the other hand the aimed use of light is often successful when treating a number of mental and psychological disorders. In particular, bright light therapy (BLT) showed positive results with respect to seasonal affective disorders, non-seasonal depression, antepartum depression, sleep disorders, Parkinson's disease and so on. However, there is still little data concerning the effects of light therapy on functional activity of the cardiovascular system and particularly in case of hypertension. Many patients suffering from seasonal affective disorders, from different kinds of depression and so on can have some concomitant diseases, particularly hypertension of various genesis. We hypothesized that therapeutic effect of bright light may be accompanied by some changes in the biological rhythms of the cardiovascular system both in case of normal blood pressure and under hypertension.

We performed our experiments on male rats aged 32 - 34 weeks of 2 genetic strains: Wistar-Kyoto - normotensive rats and SHR - spontaneously hypertensive rats. The animals were kept in individual cages under an artificial light-dark schedule with light on at 7.00 a.m. 
and off at 7.00 p.m., 300 lux white light at eye level and nighttime absolute darkness. To estimate the effect of bright light the animals were exposed to 1 hour exposure of $\sim 10000$ lux white LED light from 10.00 to 11.00 a.m. For the analysis of daily profiles of blood pressure and heart rate we used the method of radio-telemetric monitoring which enables us to register the parameters continuously for 24 hours in totally freely moving animals. Radio-telemetry system (DSI, USA) [1] consists of a radio-transmitter (sensor) implanted in the animal body and monitoring blood pressure and ECG and sending data as a radio-signal to a receiver (nearby the cage), a computer for storing data. BP was monitored in the abdominal aorta. ECG was registered using the electrodes fixed to the animal chest along the electric axis of the heart. We began the experiments 10 days after implantation of the radio-transmitters. Blood pressure and ECG were monitored continuously for 24 hours the day before bright light treatment (control) and on the day of bright light treatment. The following parameters were estimated: mean daily (7.00 a.m. -7.00 p.m.), mean daytime (7.00 a.m. - 7.00 p.m.) and mean nighttime (7.00 a.m. - 7.00 a.m.) values of systolic blood pressure and diastolic blood pressure.

Statistical data proceeding was carried out using Statistica 6.0 (StatSoft Inc.). The mean value and the error of the mean were calculated. The significance of difference in means was determined using the Mann-Whitney $U$ test (the difference considered as significant at $p \leq 0.05)$.

In the normotensive Wistar-Kyoto rats 1 hour exposure of bright light did not induce any significant changes in the values of blood pressure and heart rate for the 24-hour period. During the time of bright light exposure (from 10.00 to 11.00 a.m.) and within the whole daytime period systolic blood pressure was significantly higher in comparison with the corresponding periods of the previous day when bright light treatment was not performed (Figure 1). However the values of diastolic blood pressure or heart rate had no significant changes compared with the controls either at the time of bright light treatment or during the whole daytime period.

In the hypertensive SHR rats the values of both systolic and diastolic blood pressure were significantly higher during bright light treatment in comparison with the same period of the previous day when bright light treatment was not performed (Figure 2, left). The mean levels of daytime (10.00 a.m. - 7.00 p.m.) systolic (but not diastolic) blood pressure was also significantly increased compared with the controls which might indicate that the effect of bright light on systolic blood pressure remains notable even after the end of its exposure (Figure 2, right). For heart rate only a tendency to an increase was seen.

An increase in blood pressure under the action of bright light might seem quite expected as it was earlier shown that exposure to stressful stimuli contributed to aggravation of hypertension $[3,5]$. However, according to our results, there was no significant elevation of heart rate either in normotensive Wistar-Kyoto or in hypertensive SHR rats which indicates the absence of true stress response. More possibly this is the activation reaction which may occur due to the stimulation of suprachiasmatic nuclei $(\mathrm{SCN})$ of the hypothalamus. This hypothesis requires further study.

\section{Conclusion}

Our data demonstrate first that in chronobiological studies we do need additional statistical and mathematical modeling methods to evaluate and describe effects of drugs (many studies, see e.g. [8] or of bright light, as used in the present study.

Secondly, we could demonstrate the application of bright light in spontaneouslyhypertensive rats, affected the level in blood pressure as well patterns in the 24-h profiles. 

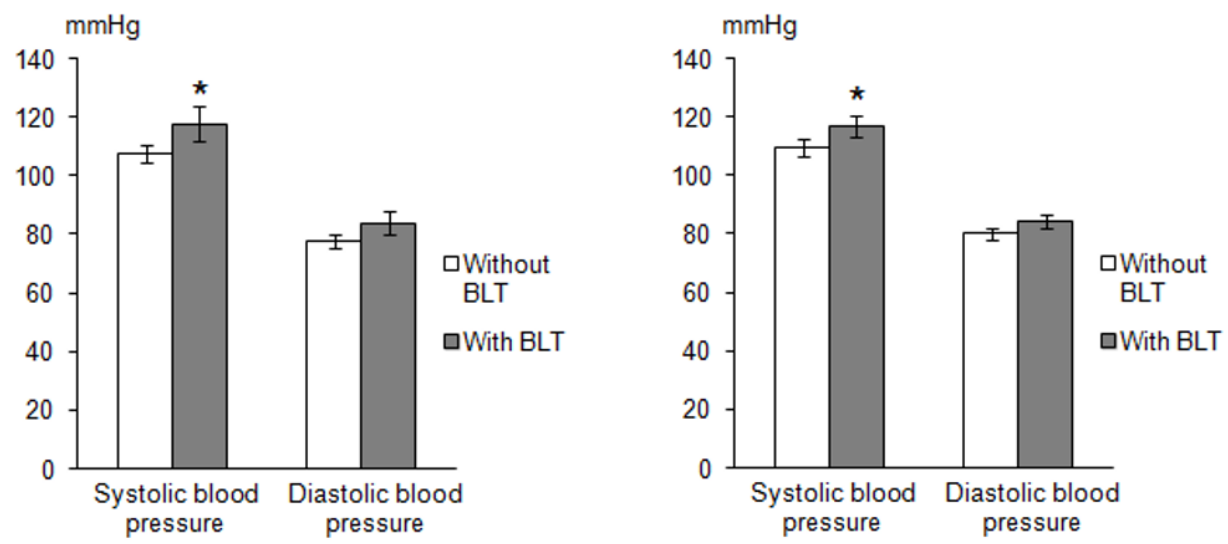

Figure 1. Left: Systolic and diastolic blood pressure in normotensive Wistar-Kyoto rats (from 10.00 to 11.00 a.m.). ${ }^{*} p \leq 0.05$ blood pressure under bright light exposure (from 10.00 to 11.00 a.m.) vs the same period of time of the previous day when bright light was not exposed. Right: Systolic and diastolic blood pressure in normotensive Wistar-Kyoto rats during the daytime period (10.00 a.m. 7.00 p.m.). ${ }^{*} p \leq 0.05$ blood pressure on the day of light exposure vs the previous day when bright light was not exposed.
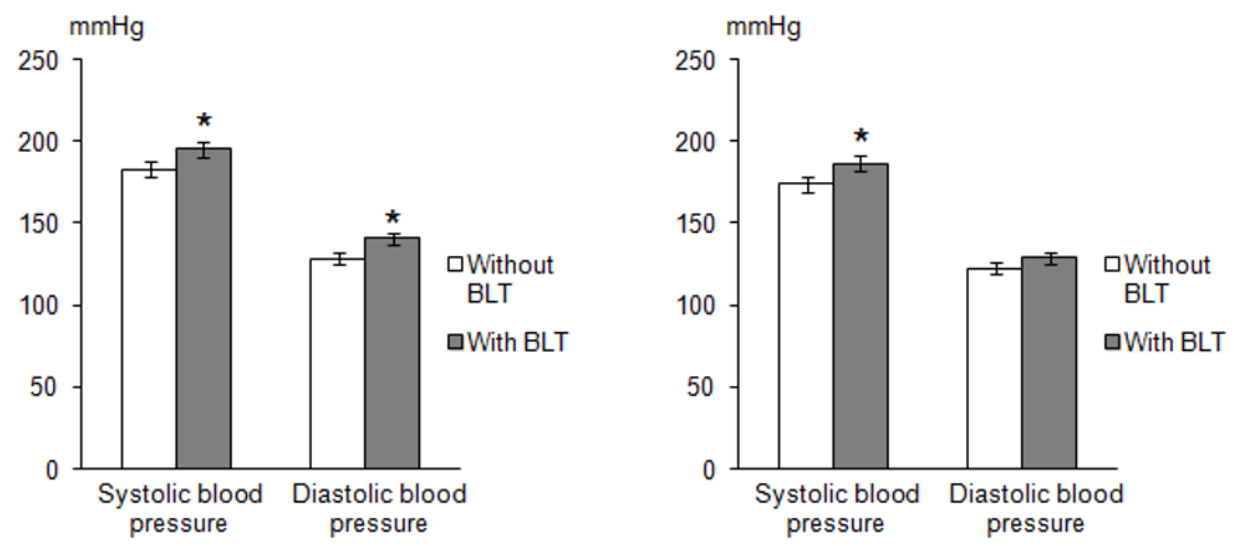

Figure 2. Left: Systolic and diastolic blood pressure in hypertensive SHR rats (from 10.00 to 11.00 a.m.). ${ }^{*} p \leq 0.05$ blood pressure under bright light exposure (from 10.00 to 11.00 a.m.) vs the same period of time of the previous day when bright light was not exposed. Right:Systolic and diastolic blood pressure in hypertensive SHR rats during the daytime period (10.00 a.m. -7.00 p.m.). ${ }^{*} p \leq 0.05$ blood pressure on the day of light exposure vs the previous day when bright light was not exposed.

These results ask for further and more detailed chronobiological studies to provide additional evidence.

Performing our chronobiological investigations with the use of continuous 24-hour telemetric monitoring for many days we obtain a very big amount of data which could be used for the prognosis of the further development of various pathological conditions, in particular hypertension. By applying the traditional statistical methods to our results we can only assess the chronostructure of some physiological functions for within the period of monitoring. 
Moreover we may miss a considerable part of such data without using contemporary methods of computer and mathematical modeling. So we could suggest that closer collaboration between chronobiology and computers sciences would give promising further results.

\section{References}

[1] Brockway B.P., Mills P.A., Azar S.H, Clin. Exp. Hypertens. A 13, 885-895 (1991)

[2] Eds. Aschoff, Jurgen, Biological Rhythms (Springer US, 1981) 563

[3] Folkow B., Hallbäck-Nordlander M., Martner J., Nordborg C., Acta Physiol. Scand., 116, 133-139 (1982)

[4] Halberg F., Int. Z. Vitaminforsch Beih 10, 225-296 (1959)

[5] Koepke J.P., Jones S., DiBona G.F., Brain Res., 404, 80-88 (1987)

[6] Lemmer B., Mattes A., Bohm M., Ganten D., Hypertension, 22, 97-101 (1993)

[7] Lemmer B., Gorbey S., Zuther P., Chronos-Fit, personal edition, 2019, Researchgate.net

[8] Lemmer B., Heart Failure Clinics, 13, $739-757$ (2017) 